DOI: $10.7242 / 2658-705 X / 2019.4 .5$

УДК 539.421

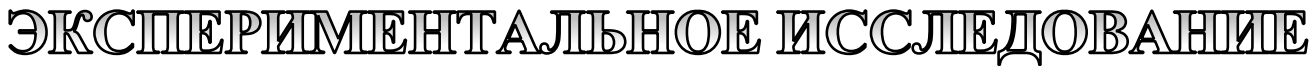

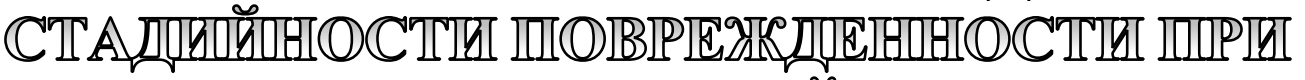

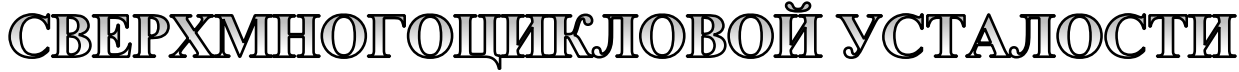

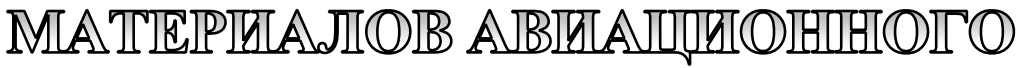 MOTOPOCTPOEIBUELAI *
}

М.В. Банников, Институт механики сплошных сред УрО РАН

И.А. Банникова, Институт механики сплошных сред УрО РАН

В.А. Оборин, Институт механики сплошных сред УрО РАН

B работе приведена методика «in situ» определения накопления необратимых усталостных повреждений, основанная на анализе нелинейных проявлений сигнала обратной связи в замкнутой системе ультразвуковой усталостной установки. В режиме сверхмногоцикловой (гигацикловой) усталости проявляются аномалии упругих свойств материала, что приводит к эффректу нелинейности в амплитуде колебаний. Эфффект возрастает с инициированием и ростом усталостной трещины. Предложенная методика была применена к образцам сплава АМГ-6 с предварительной динамической деформацией для определения момента зарождения и роста усталостной трещины. Показано, что метод применим для раннего обнаружения как поверхностной, так и для внутренней усталостной трещины. На основе широкодиапазонных определяющих соотношений для деформированного твердого тела с мезоскопическими дефектами предложена математическая модель, которая способна адекватно описывать поведение материала в процессе усталостного разрушения. Результаты численного моделирования находятся в хорошем соответствии с экспериментальными данными.

Ключевые слова: кинетика накопления дефектов, скейлинг, многочикловая усталость, гигачикловая усталость, математическое моделирование, разрушение, мезоскопические дефекты.

\section{Введение}

Определение природы зарождения усталостных трещин является одной из важнейших фундаментальных проблем для различных областей приложений, особенно если говорить о сверхмногоцикловой (гигацикловой) [1-3] усталости, когда трещина образуется внутри материала. Серии катастроф, обусловленных усталостным разрушением газотурбинных двигателей [4-6], в сочетании с высокой стоимостью оценки ресурса и потенциальной стоимостью разработки новых конструкций, стимулировали перспектив-

* Статья подготовлена при финансовой поддержке гранта РФФИ № 16-41-590892 p_a «Экспериментальное исследование стадийности поврежденности при сверхмногоцикловой усталости материалов авиационного моторостроения». 
ные концепции национальных программ в области много- и гигациклового разрушения, основанные на использовании новых фундаментальных результатов при оценке усталостной прочности [7-11].

В настоящее время после нескольких десятилетий исследований становится ясно, что усталостная поврежденность является результатом множественных многомасштабных деформаций, которые приводят к необратимым микроструктурным и топологическим изменениям [12], определяющим критические состояния поврежденности и переход к разрушению. Общие знания о механизмах циклической деформации и микроструктурных изменениях, приводящих к усталостному разрушению, позволяют связать эти критические состояния с развитием ансамбля микротрещин, зарождающихся в локализованных сдвигах.

В последние годы резко возрос интерес к фундаментальным проблемам усталости в связи с возможностью достижения усталостного ресурса, соответствующего так называемой сверхмногоцикловой (гигацикловой) усталости [1-10]. Результаты этих исследований поставили, в частности, вопрос о существовании предела усталости при переходе от многок гигацикловым условиям нагружения.

Развитие повреждений при усталости традиционно связывается с микропластическими деформациями [1-2], которые формируются в условиях циклического нагружения, инициирующего различные микроструктурные механизмы, контролирующие долговечность и зависящие от природы и исходной структуры материала. Для пластичных металлов наблюдается специальный вид усталостной (циклической) локализации деформации (persistent slip bands - PSB), развитие которой традиционно ведет к инициированию трещин в приповерхностной зоне. Вторым распространенным случаем, например, при усталостном разрушении высокопрочных сталей, является зарождение повреждений (микротрещин) в окрестности включений при низких амплиту- дах нагрузки, соответствующих сверхмногоцикловой усталости (СМУ).

Характерной чертой развития разрушения в условиях гигацикловой усталости является решающее влияние на усталостную долговечность стадии инициирования усталостной трещины. При этом качественным отличием является образование усталостной трещины в объеме материала, что решающим образом меняет постановку проблемы оценки усталостного ресурса, методов исследования стадийности развития разрушения. В противоположность сложившимся традициям в области многоцикловой усталости, где центральное внимание уделяется стадии распространения трещин, возникает фундаментальная проблема о зарождении усталостной трещины в ходе многомасштабных процессов развития поврежденности, ассоциируемой с дефектами различной природы (включения, полосы локализованного пластического сдвига, микротрещины, поры). В [12-13] отмечается, что стадийность разрушения характеризуется эффектами «необратимости», инициированными формированием локализованных сдвигов, играющих ключевую роль при зарождении усталостной трещины, что может проявляться в признаках нелинейности упругого поведения материалов, «аномалий упругой податливости» усталостных образцов. Роль стадии инициирования особенно важна для гигацикловых режимов нагружения, которые характеризуются зарождением очага разрушения в форме «fish-еуе» («рыбий глаз») в объеме материала [13-15].

Химический состав сплава АМг-6 представлен в таблице 1, геометрия образцов изображена на рис. 1.

Перед усталостными испытаниями материал, из которого впоследствии вырезались образцы, был подвержен предвари-

Таблица 1

Химический состав АМг-6

(в процентном содержании)

\begin{tabular}{|c|c|c|c|c|c|c|c|c|}
\hline $\mathrm{Al}$ & $\mathrm{Cu}$ & $\mathrm{Mg}$ & $\mathrm{Mn}$ & $\mathrm{Si}$ & $\mathrm{Fe}$ & $\mathrm{Zn}$ & $\mathrm{Be}$ & $\mathrm{Ti}$ \\
\hline $91,1-$ & 0,10 & $5,8-$ & $0,5-$ & 0,4 & 0,4 & 0,20 & $0,0002-$ & $0,02-$ \\
93,68 & & 6,8 & 0,8 & & & & 0,005 & 0,1 \\
\hline
\end{tabular}




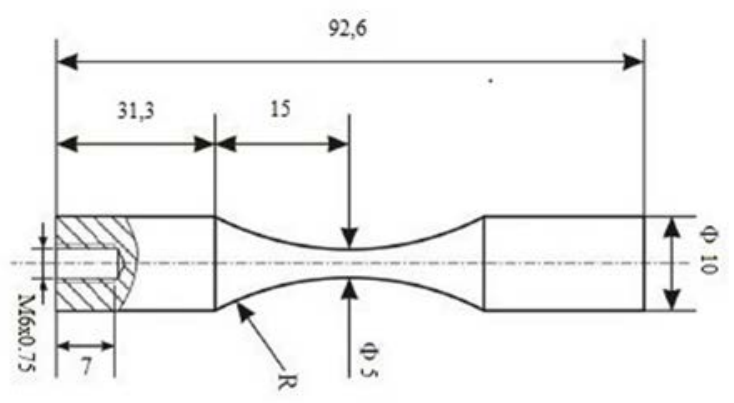

Рис.1. Геометрия образциов

тельному динамическому деформированию. Основа толщиной 15 мм подвергалась ударному нагружению алюминиевой пластиной толщиной 4 мм и диаметром 120 мм, разогнанной с помощью плосковолнового взрывного генератора до скорости $\sim 1400 \mathrm{M} / \mathrm{c}$ в лаборатории реологических свойств конденсированных сред при импульсных воздействиях Института проблем химической физики РАН [16].

Усталостное нагружение проводилось на ультразвуковой [13] испытательной машине резонансного типа Shimadzu USF2000 (рис. 2) при уровнях напряжений 90162 МПа и симметричном цикле $\mathrm{R}=-1$. Принцип работы испытательной машины заключается в том, что в системе «генера-

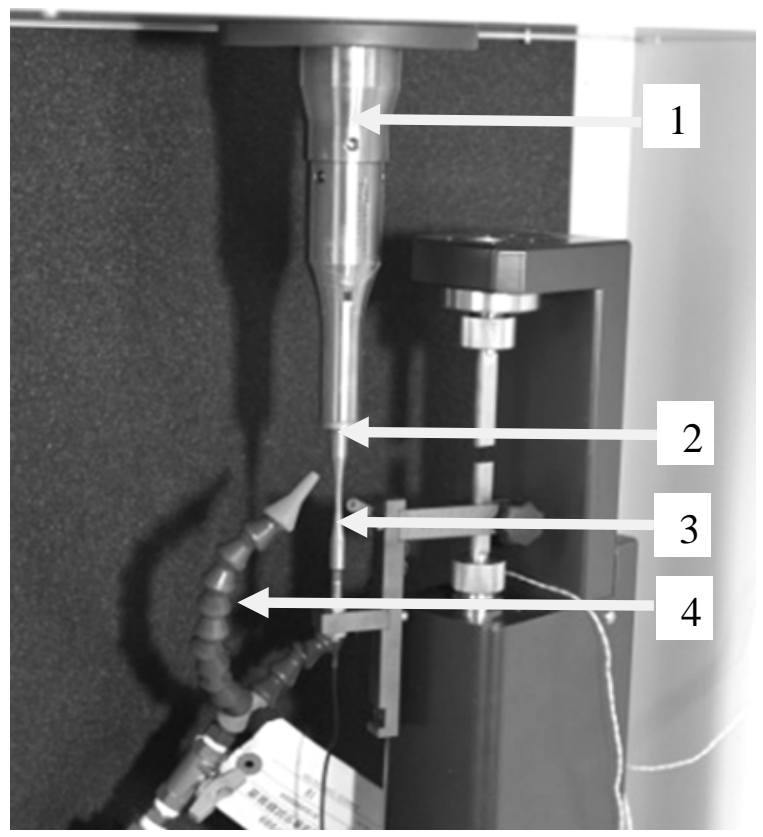

Рис. 2. Внешний вид экспериментальной установки: 1 - волновод, 2 - образец, 3 - датчик перемещения, 4-система охлаждения тор-волновод-образец» образуется стоячая волна. При этом максимум амплитуды перемещений приходится на торцы образца, а максимум амплитуды напряжений - на его центр. Со стороны свободного торца образца закреплен индуктивный датчик, позволяющий определять перемещения торца с точностью десятых долей микрон и частотой до 1 МГц. Машина позволяет испытывать материалы на базе $10^{9}-10^{10}$ циклов с амплитудой от 1 и до нескольких десятков микрон с частотой 20 кГц, что сокращает время испытания до нескольких дней в отличие от классических усталостных установок, на которых такое число циклов достигается за годы испытаний.

\section{Использование нелинейной акустики для неразрушающего контроля усталостного разрушения}

Метод основан на инициировании продольного конечно-амплитудного возмущения $A_{0}$ с частотой $\omega_{0}$ с одной стороны образца, тогда как другой его торец остается свободным. Колебания свободного торца образца будут содержать ряд гармонических составляющих: компоненты с амплитудой $A_{1}$ на основной частоте $\omega_{0}$, амплитудой $A_{2}$ второй гармоники частотой $2 \omega_{0}$, и так далее. Параметр нелинейности $\beta^{e}$ определяется экспериментально путем измерения абсолютных амплитуд сигналов первой $A_{1}$ и второй $A_{2}$ гармоник, соответствующих нелинейному закону упругости:

$$
\begin{aligned}
& \sigma=A_{2}^{e}\left(\frac{\partial u}{\partial a}\right)+\left(\frac{1}{2}\right) A_{3}^{e}\left(\frac{\partial u}{\partial a}\right)^{2}+\ldots= \\
& =A_{2}^{e}\left[\left(\frac{\partial u}{\partial a}\right)-\frac{1}{2} \beta^{e}\left(\frac{\partial u}{\partial a}\right)^{2}+\ldots\right],
\end{aligned}
$$

где $\sigma$ - нагрузка, $u$ - перемещение, $a$ пространственная координата, $A_{2}^{e}$ и $A_{3}^{e}-$ упругие коэффициенты второго и третьего порядка соответственно. Введением коэффициента нелинейности $\beta^{e}=-\left(A_{3}^{e} / A_{2}^{e}\right)$ волновое уравнение может быть представлено в виде

$$
\frac{\partial^{2} u}{\partial t^{2}}=c^{2}\left[1-\beta^{e}\left(\frac{\partial u}{\partial a}\right)\right]\left(\frac{\partial^{2} u}{\partial a^{2}}\right),
$$


где $u$ - компонента вектора смещений в направлении $a, c$ - продольная скорость звука, $t$ - время. Его решение, учитывая, что возмущение торца $u=u_{1} \cos (\omega t)$, будет иметь вид

$$
u=u_{0}+u_{1} \cos (\omega t)+u_{2} \sin 2(\omega t-k a)+\ldots,
$$

где $u_{2}=(1 / 8) k^{2} \beta^{e} u_{1}^{2} a, k=\omega_{0} / v$ - волновое число. Откуда можно выразить:

$$
\beta^{e}=8 u_{2} / k^{2} u_{1}^{2} a .
$$

При исследовании нелинейных явлений в режиме гигацикловой усталости с помощью измерения амплитуд основной и второй гармоник определяется относительный параметр:

$$
\beta_{\text {relative }}=\beta / \beta_{0},
$$

где $\beta_{0}$ относится к неповрежденному материалу.

\section{Измерение коэффициента нелинейности}

Для определения коэффициента нелинейности прологарифмируем обе части уравнения (1.1):

$$
\log (\beta)=\log (k)+\log \left(u_{2}\right)-2 \log \left(u_{1}\right)
$$

Амплитуда колебаний измеряется в децибелах в масштабах $A_{1}=20 \log \left(u_{1}\right)$ и $A_{2}=20 \log \left(u_{2}\right)$, тогда соотношение принимает вид:

$20 \log (\beta)=20 \log (k)+20 \log \left(u_{2}\right)-40 \log \left(u_{1}\right)$,

$$
20 \log (\beta)=K+A_{2}-2 A_{1} \text {. }
$$

Таким образом, относительный параметр нелинейности в можно найти из формулы

$$
20 \log \left(\beta / \beta_{0}\right)=\left(A_{2}-2 A_{1}\right)-\left(A_{2}-2 A_{1}\right)_{0} .
$$

Приведены зависимости коэффициента нелинейности ßrelative от амплитуды нагружения в течение усталостной долговечности для алюминиевого сплава АМг-6. В процессе эксперимента Brelative незначительно увеличивается на начальном этапе, что обычно связывают с эффектом нагрева образца и, вследствие этого, изменением его линейных размеров [17-19], затем основную часть времени эксперимента амплитуда второй гармоники остается постоянной и значительно увеличивается при образовании усталостной трещины и её росте (рис. 3, a). Амплитуда второй гармоники связывается с деформацией, вызванной системой дефектов. При задаваемых параметрах нагружения макроскопически система находится в упругой области, и все деформации являются обратимыми. Дефекты слабо коррелируют между собой, а амплитуда второй гармоники мала и слабо изменяется, однако в процессе многочисленных циклических сдвигов образуются необратимые повреждения, которые начинают взаимодействовать друг с другом, что приводит впоследствии к образованию усталостной трещины.

У одного из образцов, разрушенного с образованием поверхностной трещины, было обнаружено нехарактерное для других монотонное снижение амплитуды второй гармоники в процессе испытаний (рис.3.б). Предполагается, это связано с тем, что изначальная структура материала была насыщена дефектами, поскольку образцы были предварительно деформи-

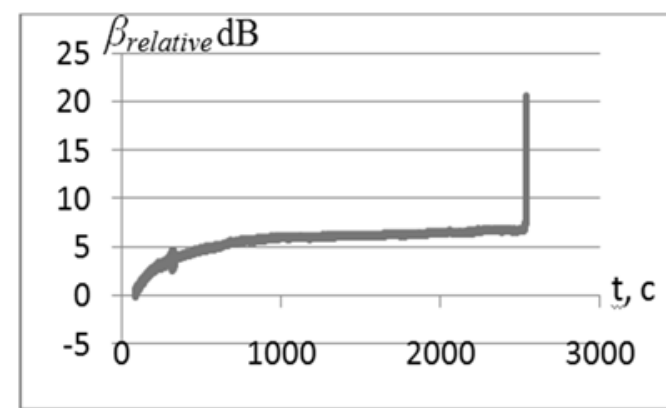

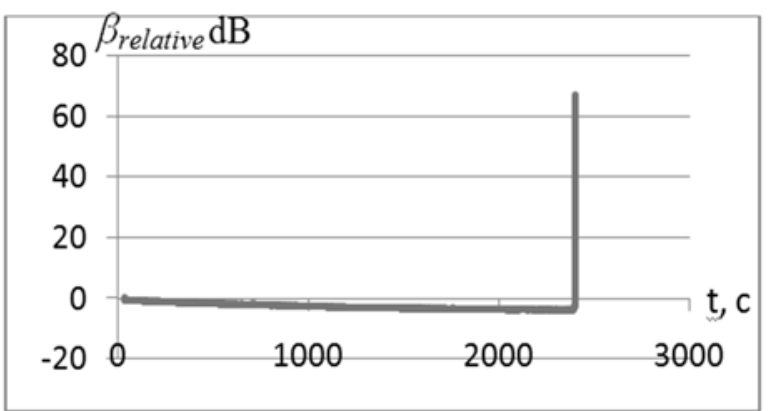

$\sigma$

Pис.3 Изменение показателя нелинейности Brelative $(d B)$ в прочессе испытаний у образиа с внутреннй трещчиной $а-\sigma=138 \mathrm{MPa}, N=7,51 \cdot 10^{8}$ (Fish-еуе) и образиа с поверхностной трещчиной (б) $\sigma=140 \mathrm{MPa}, N=2,72 \cdot 10^{8}$ 
рованы. В процессе циклического нагружения малой амплитудой в материале происходила релаксация внутренних напряжений [20], что приводило к уменьшению нелинейности сигнала. При выработке ресурса релаксации напряжений наблюдается лавинообразный рост амплитуды второй гармоники, что свидетельствует о высокой корреляции дефектов и их взаимодействии на больших масштабах по сравнению с внутренним зарождением усталостной трещины.

На рис. 4. показан рост амплитуды второй гармоники в процессе роста усталостной трещины. В момент времени $5,415 \cdot 10^{\wedge} 4$ программное обеспечение остановило эксперимент на основании значительного увеличения амплитуды второй гармоники (в 6 раз), что свидетельствовало об образовании усталостной трещины. В дальнейшем эксперимент был запущен снова, что позволило наблюдать, как амплитуда второй гармоники увеличивается с ростом длины трещины. Таким образом, параметр ßrelative можно использовать не только как индикатор появления усталостной трещины, но и определять по нему её размер, как это было сделано в работе [21].

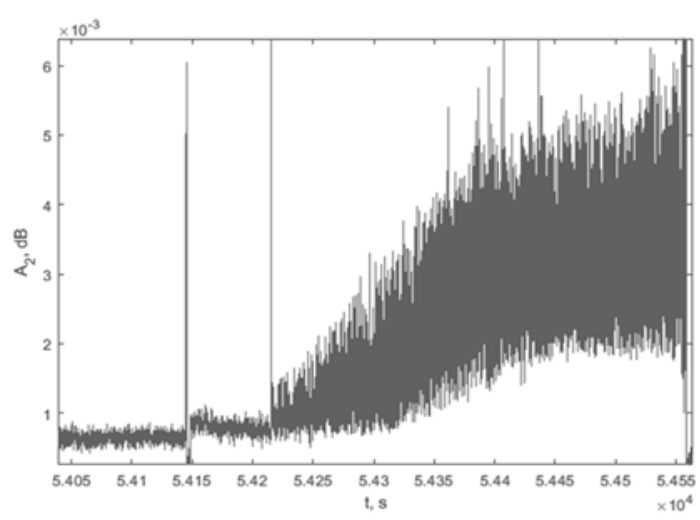

Рис 4. Амплитуда второй гармоники образца АМГ-6, предварительно подвергнутого ударно-волновому нагружению. Разница по количеству ииклов между моментом обнаружения зарождения усталостной трешины и выходом её на макроскопическое разрушение (вывод системы из резонанса) составила более $2 \cdot 10^{6}$ ичиклов

\section{Математическая модель}

Широкодиапазонные определяющие соотношения [22-24], описывающие связь кинетики поврежденности, обусловленной дефектами, с релаксационными свойствами материалов, использованы для построения математической модели, способной описывать деформационное поведение металлов и сплавов при усталостном нагружении.

Идентификация параметров построенной модели проводилась в одноосном случае:

$$
\begin{gathered}
\dot{\sigma}=\lambda \dot{\varepsilon}+2 G\left(\dot{\varepsilon}-\dot{\varepsilon}^{p}-\dot{p}\right), \\
\dot{\varepsilon}^{p}=\dot{\varepsilon}_{0}^{n_{\varepsilon}}\left(\Gamma_{\sigma} \sigma-\Gamma_{p \sigma} \frac{\partial F}{\partial p}\right), \\
\dot{p}=\dot{\varepsilon}_{0}^{n_{p}}\left(\Gamma_{p \sigma} \sigma-\Gamma_{p} \frac{\partial F}{\partial p}\right), \\
\dot{\delta}=-\dot{p}_{0}^{n_{\delta}} \Gamma_{\delta} \frac{\partial F}{\partial \delta}, \\
\frac{F}{F_{m}}=\frac{p^{2}}{2}-\frac{p^{2}}{2 \delta}+c_{1} p+ \\
+c_{2} \ln \left(c_{3}+c_{4} p+p^{2}\right)-\frac{\sigma p}{2 G},
\end{gathered}
$$

где $\dot{\varepsilon}, \sigma, \dot{\varepsilon}^{p l}, \mathrm{p}$ - соответствующие компоненты тензоров $\dot{\boldsymbol{\varepsilon}}, \boldsymbol{\sigma}, \dot{\boldsymbol{\varepsilon}}^{p}, \mathbf{p}$.

На первом этапе идентификации решается задача минимизации невязки между экспериментальной и расчётной диаграммами деформирования. При этом определяются параметры $\underline{\Gamma}_{\sigma}, \Gamma_{p \sigma}, \Gamma_{p}$, безразмерная скорость деформации $\dot{\varepsilon}_{0}=1$ (статическое нагружение), $\delta=$ const $=1,15$.

На втором этапе с использованием известных значений $\underline{\Gamma_{\sigma}}, \Gamma_{p \sigma}, \Gamma_{p}$, решается задача минимизации невязки между экспериментальной и расчётной диаграммами деформирования при различных скоростях деформации. При этом определяются параметры $n_{\varepsilon}$ и $n_{p}, \delta=$ const $=1,15$. Иллюстрация решения задач оптимизации представлена в табл. 2.

Таблий 2

Предел текучести в эксперименте [25] и расчёте при различных скоростях деформации

\begin{tabular}{|c|c|c|}
\hline $\begin{array}{c}\text { Скорость } \\
\mathrm{c}^{-1}\end{array}$ & $\begin{array}{c}\text { Предел текучести, } \\
\text { МПа } \\
\text { (эксперимент, } \\
[25,26])\end{array}$ & $\begin{array}{c}\text { Предел } \\
\text { текучести, } \\
\text { МПа (расчёт) }\end{array}$ \\
\hline 0,0001 & 165 & 166 \\
\hline 520 & 175 & 176 \\
\hline 1210 & 210 & 213 \\
\hline
\end{tabular}


Завершающий этап идентификации параметров модели заключается в определении значений $\Gamma_{\delta}$ и $\mathrm{n}_{\delta}$, которые находятся с использованием экспериментальных данных усталостного нагружения. Выбраны две характерные точки на кривой Вёлера: (300 000 циклов, 190 МПа) и (300 000000 циклов, 156 МПа). Параметры $\Gamma_{\delta}$ и $\mathrm{n}_{\delta}$ выбирались таким образом, чтобы при амплитуде нагружения 190 МПа материал выдерживал 300000 циклов до разрушения, а при $156 \mathrm{MПа} \mathrm{-} 300000000$ циклов соответственно. Частота нагружения при этом равнялась 20000 Гц, как и в эксперименте.

Таким образом, полный набор констант для сплава АМг-6 следующий:

Константы, известные из литературы [26]: $\quad \rho=2670 \kappa г / \mathrm{m}^{3}, \quad \lambda=41$ ГПа, $G=27$ ГПа. Определённые константы: $\underline{\Gamma}_{\sigma}=529(\Pi a \cdot c)^{-1}, \quad \Gamma_{p \sigma}=38,5(\Pi a \cdot c)^{-1}$, $\Gamma_{p}=2,9(\Pi a \cdot c)^{-1}, \quad \Gamma_{\delta}=3,46(\Pi a \cdot c)^{-1}$, $n_{\varepsilon}=n_{p}=0,967, \mathrm{n}_{\delta}=1,985$.

\section{Результаты численного моделирования}

Численное исследование поведения реальной конструкции, и даже лабораторного образца, в режиме гигацикловой усталости не представляется возможным в связи с требованием огромных вычислительных и временных ресурсов. Поэтому вместо решения краевой задачи рассматривается задача нагружения представительного объёма материала (2.1)-(2.5) с условиями нагружения в виде приложенных циклических напряжений (2.6) с амплитудой $\varepsilon_{A}$ и частотой $v=20000$ Гц, а также начальными условиями (2.7):

$$
\begin{gathered}
\sigma(t)=\sigma_{A} \sin (2 \pi v t), \\
\left.p\right|_{t=0}=0,\left.\delta\right|_{t=0}=\delta_{0}=1,15 .
\end{gathered}
$$

Условие нагружения (19) можно записать в терминах деформаций:

$$
\varepsilon(t)=\varepsilon\left(\sigma_{A}\right) \sin (2 \pi v t),
$$

где $\varepsilon(\sigma)$ - функция, переводящая напряжения в деформации для сплава АМг-6. Продифференцировав (2.8) по времени, получим

$$
\dot{\varepsilon}(t)=\varepsilon\left(\sigma_{A}\right) 2 \pi v \cos (2 \pi v t) .
$$

В эксперименте условия нагружения задаются в виде (2.6), но для численного расчёта удобнее использовать выражение (2.9), которое математически эквивалентно (2.8). Численно построенная кривая Вёлера и её сопоставление с экспериментальными данными представлены на рис. 5.

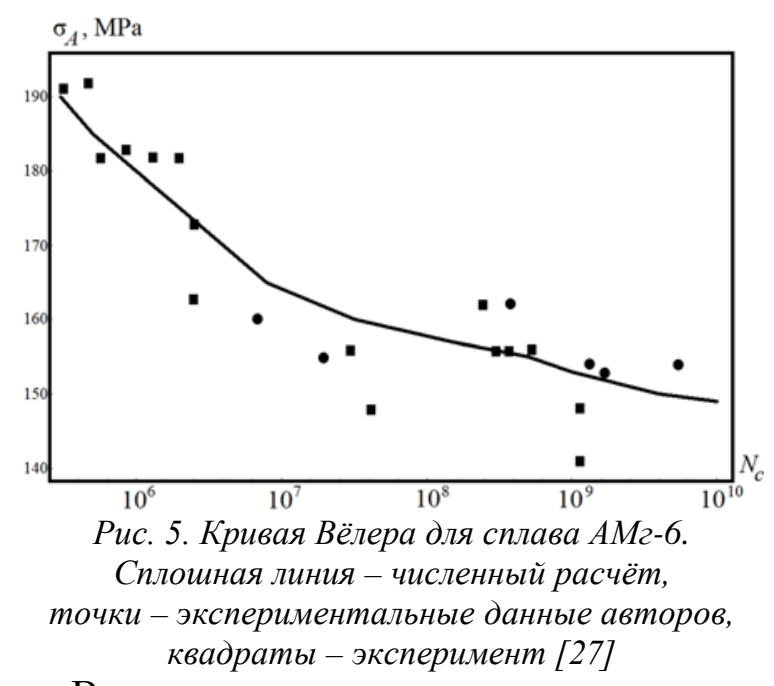

Ввиду амплитуд прикладываемых напряжений ниже предела текучести макроскопически материал находится в упругой области, а прикладываемые деформации можно считать малыми. Тогда выражение (2.1) можно переписать в виде

$$
\sigma=\lambda \varepsilon+2 G\left(\varepsilon-\varepsilon^{p}-p\right) .
$$

Так как макропластической деформации не наблюдается, то в режиме гигацикловой усталости за накопление поврежденности и разрушение отвечает параметр $p$. Рост дефектов приводит к постепенному изменению амплитуды приложенных напряжений, а следовательно, и к амплитуде приложенных перемещений, так как для $\sigma_{A}$ всегда ставится в соответствие некоторая амплитуда перемещений $\mathrm{U}_{A}$ в нагружающей машине:

$$
\sigma_{A}=\lambda \varepsilon\left(\sigma_{A}\right)+2 G\left(\varepsilon\left(\sigma_{A}\right)-p\right)
$$

После достижения параметра $p$ некоторого критического значения $p_{c}$ (которое соответствует $\delta_{f} \approx 0.4$, происходит лавинообразный рост дефектов, приводящий к разрушению материала, что в эксперименте соответствует лавинообразному изменению амплитуды второй гармоники перемещений (рис 6.). На рис. 6 изображены нормированные кривые изменения параметра $p$, отнесённого к $p_{c}$, и амплитуда второй гармоники перемещений, отнесённая к её 


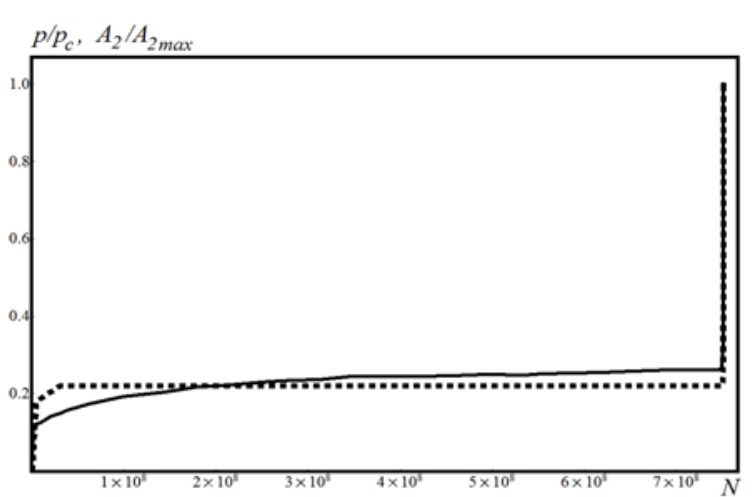

Рис.6. Кинетика дефектов (пунктирная линия) в расчёте и изменение амплитуды второй гармоники перемещуений в эксперименте (сплошная линия)

максимальному значению; представлены экспериментальные данные, соответствующие предварительно нагруженному образцу. Согласно предложенной модели, изменение структуры за счёт предварительного нагружения описывается в терминах параметра $\delta$, а именно: предварительно нагруженному образцу соответствует меньшее значение начального условия $\delta_{0}$. Для получения пунктирной кривой (см. рис. 6) решалась задача $(2.1-2.6,2.7,2.9)$ с начальным условием $\delta_{0}=1,02$.

Таким образом, предложенная модель способна адекватно описывать поведение материала в режимах много- и гигацикловой усталости. Опираясь на результаты численного моделирования, можно сделать вывод о том, что изменение амплитуды второй гармоники перемещений в эксперименте даёт адекватную информацию о накоплении повреждённости в материале.

\section{Заключение}

К основным результатам выполнения проекта можно отнести следующее:

1. Разработан и собран комплекс, включающий ультразвуковую испытательную машину Shimadzu USF-2000 и систему сбора и анализа данных колебаний образцов, для реализации экспериментальной программы по исследованию стадийности внутреннего зарождения и роста усталостной трещины.

2. Анализ сигнала, полученный с датчика перемещения свободного торца, показал увеличение второй гармоники колебаний вблизи критического разрушения материала, что может послужить критерием при оценке долговечности материалов и конструкций.

3. Показано, что чувствительность метода определения момента зарождения трещины по коэффициенту нелинейности превышает способ определения по резонансной частоте. Чувствительность метода зависит как от внутренней структуры материала (более дефектные структуры демонстрируют более раннее проявление нелинейных сигналов), так и от геометрии материала (на образцах с большим внутренним сечением чувствительность методики уменьшается по сравнению с образцами с меньшим сечением)

4. При циклическом нагружении алюминия, подвергнутого предварительному динамическом нагружению, было обнаружено уменьшение коэффициента нелинейности в процессе испытаний до определенного момента времени и его резкий рост непосредственно перед разрушением. В работе [20] теоретическим и экспериментальным методами исследовалось влияние ультразвуковой обработки на структуру и свойства ультрамелкозернистых металлов, полученных с помощью интенсивной пластической деформацией. Им было показано, что циклическое нагружение ультразвуком знакопеременной нагрузкой низкой амплитуды повышает подвижность дислокаций, что приводит к релаксации внутренних деформаций и напряжений. Возможно, именно с этим эффектом связано первичное уменьшение коэффициента нелинейности, но в момент, когда механизмы релаксации внутренних деформаций иссякают, происходит её локализация и зарождается внутренняя трещина, что приводит к резкому росту коэффициента нелинейности.

5. Разработана система обратной связи, которая, анализируя текущий сигнал со средними показателями, позволяет своевременно установить увеличение второй гармоники и сохранить образец с имеющимся в нем зародышем усталостной трещины. Система чувствительна к образцам с изначально высокой степенью поврежден- 
ности, например, предварительно деформированным (в рамках проекта исследовались предвартиельное динамическое и ударно-волновое нагружение). Таким образом, методика может быть использована для контроля соответствия нормативным показателям изделий из металлов, работающих в сверхмногоцикловом диапазоне. Образцы с имеющимися внутренними трещинами, полученные с помощью разработанной методики, в дальнейших работах планируется исследовать методами рентгеновской томографии, что позволит развить фундаментальные аспекты зарождения и распространения усталостных трещин.

На основе кинематической модели нагружения Кельвина разработана математическая модель развития поврежденности и её влияния на амплитуду колебаний второй гармоники. Полученная модель находится в качественном соответствии с экспериментальными данными.

\section{Библиографический список}

1. Ботвина Л.Р. Гигацикловая усталость - новая проблема физики и механики разрушения // Заводская лаборатория. Диагностика материалов. - 2004. - Т. 70. - № 4. - С. 41.

2. Bathias C., Paris P.C. Gigacycle Fatigue in Mechanical Practice. - Marcel Dekker Publisher Co, 2005. - 328 p.

3. Оборин B.A., Банников M.В., Наймарк О.Б., Palin-Luc T. Масштабная инвариантность роста усталостной трещины при гигацикловом режиме нагружения // Письма в журнал технической физики. - 2010. - Т. 36. -Вып. 22. - C. 76-82. DOI: https://doi.org/10.1134/S106378501011026X.

4. Cowles B.A. High cycle fatigue in aircraft gas turbines - an industry perspective // International Journal of Fracture, - 1996. - Vol. 80. - P. 147-163. DOI: https://doi.org/10.1007/BF00012667.

5. Шанявский А.А. Моделирование усталостных разрушений металлов. Синергетика в авиации. Уфа: ООО «Монография», 2007. - 500 с.

6. Nicholas T. High Cycle Fatigue. A Mechanics of Material Perspective. - Elsevier, - 2006. - 641 p.

7. Peters J.O., Ritchie R.O. Influence of foreign object damage on crack initiation and early crack growth during high-cycle fatigue of Ti-6Al-4V, // Eng. Fract. Mech, - 2006. - Vol. 67. - P. 193-207.

8. Spanrad S., Tong J. Characterisation of foreign object damage (FOD) and early fatigue crack growth in laser shock peened Ti-6Al-4V aerofoil specimens // Materials Science and Engineering A, - 2011. Vol. 528. - P. 2128-2136. DOI: https://doi.org/10.1016/j.msea.2010.11.045.

9. Oakley S.Y., Nowell D. Prediction of the combined high- and low-cycle fatigue performance of gas turbine blades after foreign object damage // International Journal of Fatigue, - 2007. - Vol. 29. - P. 69-80. DOI: https://doi.org/10.1016/j.ijfatigue.2006.02.042.

10. Chen Xi. Foreign object damage on the leading edge of a thin blade // Mechanics of Materials, - 2005. Vol. 37. - P. 447-457. DOI: https://doi.org/10.1016/j.mechmat.2004.03.005.

11. Nowell D., Duó P., Stewart I.F. Prediction of fatigue performance in gas turbine blades after foreign object damage // International Journal of Fatigue, - 2003. - Vol. 25. - P. 963-969. DOI: https://doi.org/10.1016/S0142-1123(03)00160-9.

12. Mughrabi $H$. Microstructural mechanisms of cyclic deformation, fatigue crack initiation and early crack growth // Philosophical Transactions of the Royal Society A: Mathematical, Physical and Engineering Sciences, - 2015. - Vol. 373(2038). 20140132. DOI: https://doi.org/10.1098/rsta.2014.0132.

13. Zhang, $\mathrm{Li}-\mathrm{Li}$, [et al.] On the formation mechanisms of fine granular area (FGA) on the fracture surface for high strength steels in the VHCF regime // International Journal of Fatigue, - 2016. - Vol. 82. P. 402 -410. DOI: 10.1016/j.ijfatigue.2015.08.021.

14. Oborin V., Sokovikov M., Bilalo D., \& Naimark O. Multiscale study of morphology of the fracture surface aluminum-magnesium alloy with consecutive dynamic and gigacycle loading // Procedia Structural Integrity, - 2016. - Vol. 2. - P. 1063-1070. DOI: https://doi.org/10.1016/j.prostr.2016.06.136.

15. Froustey C., Naimark O., Bannikov M., Oborin V. Microstructure scaling properties and fatigue resistance of pre-strained aluminium alloys (part 1: AlCu alloy // European Journal of Mechanics A/Solids, - 2010. Vol. 29. - P. 1008-1014. DOI: https://doi.org/10.1016/j.euromechsol.2010.07.005.

16. Oborin V.A., Bayandin Yu. V., Bilalov D. A., Sokovikov M. A., Chudinov V. V, Naimark O. B. Self-similar laws of damage development and evaluation of the reliability of alloys D16T and AMg6 under combined dynamic and gigacycle loading // Phys. Mezomekh, - 2018. - Vol. 21(6). - P. 135-145 DOI: $10.1134 /$ S1029959919020048.

17. John H. Cantrell, William T. Yost Nonlinear ultrasonic characterization of fatigue microstructures // Int. J. of Fatigue, - 2001. - Vol. 23. - P. 487-490. DOI: https://doi.org/10.1016/S0142-1123(01)00162-1.

18. Kumar A., Torbet J.C. Pollock M.T., Jones W.J. In situ characterization of fatigue damage evolution in a cast Al alloy via nonlinear ultrasonic measurements // ActaMaterialia, - 2010. - Vol. 58(6). P. 2143-2154. DOI: https://doi.org/10.1016/j.actamat.2009.11.055. 
19. Kumar A. [et al.] In situ damage assessment in a cast magnesium alloy during very high cycle fatigue // ScriptaMaterialia, - 2011. - Vol. 64(1). - P.65-68. DOI: https://doi.org/10.1016/j.scriptamat.2010.09.008.

20. Назаров A.A. Неравновесные границы зерен в объемных наноструктурных материалах и их возврат под влиянием нагрева и циклической деформации. Обзор // Письма о материалах, - 2018. - Т. 8. - № 3. c. 372-381 DOI: $10.22226 / 2410-3535-2018-3-372-381$.

21. Li W., Cui H., Wen W., Su X., C.C. Engler-Pinto Jr.: In situ Nonlinear Ultrasonic for Very High Cycle Fatigue Damage Characterization of a Cast Aluminum Alloy // Materials Science and Engineering A, 2011. - Vol. 645. P. 248-254. DOI: https://doi.org/10.1016/j.msea.2015.08.029.

22. Билалов Д.А., Баяндин Ю.В., Наймарк О.Б. Математическое моделирование процесса разрушения сплава АМг2.5 в режиме много- и гигацикловой усталости // Вычислительная механика, - 2018. T. 11. - № 3. - c. 323-334. DOI: https://doi.org/10.7242/1999-6691/2018.11.3.24.

23. Naimark O.B. Collective properties of defects ensembles and some nonlinear problems of plasticity and fracture // Physical mesomechanics, - 2003. - Vol. 6(4), P. 39-63.

24. Bannikov M., Bilalov D., Oborin V., Naimark O. Damage evolution in the AMg6 alloy during high and very high cycle fatigue, Frattura ed Integrità Strutturale, - 2019. - Vol. 49. - P. 383-395; DOI: 10.3221/IGF-ESIS.49.38.

25. Glushak B.L., Ignatova O.N., Pushkov V.A., Novikov S.A., Girin A.S., Sinitsyn V.A. Dynamic Deformation of Aluminum Alloy AMg-6 at Normal and Higher Temperatures, Journal of Applied Mechanics and Technical Physics., - 2000. - Vol. 41(6). - P. 1083-1086. DOI: https://doi.org/10.1023/A:1026662824249.

26. Frolov K.V. Mechanical Engineering. Encyclopedia. Volume II-3: Non-ferrous metals and alloys. Composite metallic materials. - Moscow, Mechanical Engineering, - 2001.- 880 p.

27. Яковлева Т.Ю., Матохнюк Л.Е. Прогнозирование характеристик сопротивления усталости металлов при различных частотах нагружения // Проблемы прочности, -2004. - № 4. - С. 145-155.

\title{
EXPERIMENTAL STUDY OF DAMAGE ACCUMULATION STAGING IN GIGACYCLE FATIGUE OF AVIATION MOTORS MATERIALS
}

\author{
M.V. Bannikov, I.A. Bannikova, V.A. Oborin \\ Institute of Continuum Media Mechanics UB RAS
}

Paper presents the «in situ» method for determining irreversible fatigue damage accumulation based on the analysis of nonlinear manifestations of the feedback signal in a closed system of an ultrasonic fatigue machine. During very high cycle (gigacycle) fatigue, the anomalies appear of the material elastic properties appear, which leads to a nonlinearity effect in the amplitude of oscillations. This effect increases with the initiation and growth of fatigue cracks. The technology was applied to samples of AlMG-6 alloy with preliminary dynamic deformation to determine the moment of initiation and growth of the fatigue crack in very high cycle fatigue regime. This method is applicable for the early detection of fatigue cracks both on the surface, and inside the material under cyclic loading in the ultrasonic mode. On the basis of wide-range defining relations for a deformable solid body with mesoscopic defects, a mathematical model has been proposed that can adequately describe behavior of the material during fatigue failure. The results of mathematical modeling are in good agreement with the experimental data.

Keywords: damage accumulation kinetics, scaling, high- and very high cycle fatigue, mathematical modeling, destruction, mesoscopic defects.

\section{Сведения об авторах}

Банников Михаил Владимирович, кандидат физико-математических наук, научный сотрудник лаборатории Физических основ прочности Института механики сплошных сред УрО РАН (ИМСС УрО РАН), 614013, г. Пермь, ул. Академика Королева, 1; e-mail: mbannikov@icmm.ru

Банникова Ирина Анатольевна, кандидат физико-математических наук, сотрудник лаборатории Физических основ прочности, ИМСС УрО РАН; e-mail: mbannikov@icmm.ru

Оборин Владимир Александрович, ведущий инженер лаборатории Физических основ прочности, ИМСС УpO PAН; e-mail: oborin@icmm.ru 